\title{
Impact of coronary collateralization on long-term clinical outcomes in type 2 diabetic patients after successful recanalization of chronic total occlusion
}

\section{Zhen Kun Yang}

Department of Cardiology,Rui Jin Hospital, Shanghai Jiao Tong University School of Medicine

\section{Ying Shen}

Department of Cardiology, Rui Jin Hospital, Shanghai Jiao Tong University School of Medicine

\section{Yang Dai}

Institute of Cardiovascular Disease, Shanghai Jiao Tong University School of Medicine

\section{Xiao Qun Wang}

Department of Cardiology,Rui Jin Hospital, Shanghai Jiao Tong University School of Medicine

Jian Hu

Department of Cardiology, Rui Jin Hospital,Shanghai Jiao Tong University

\section{Feng Hua Ding}

Department of Cardiology, Rui Jin Hospital, Shanghai Jiao Tong University

\section{Rui Yan Zhang}

Department of Cardiology, Rui Jin Hospital, Shanghai Jiao Tong University School of Medicine Lin Lu

Institute of Cardivascular Disease, Shanghai Jiao Tong University school of Medicine

Wei Feng Shen ( $\nabla$ rjshenweifeng@126.com )

Department of Cardiology, Rui Jin Hospital, Shanghai Jiao Tong University School of Medicine https://orcid.org/0000-0002-7093-2001

\section{Original investigation}

Keywords: chronic total occlusion, diabetes mellitus, coronary collateral circulation, percutaneous coronary intervention, prognosis

Posted Date: February 27th, 2020

DOI: https://doi.org/10.21203/rs.2.24761/v1

License: (c) (i) This work is licensed under a Creative Commons Attribution 4.0 International License. Read Full License 
Version of Record: A version of this preprint was published at Cardiovascular Diabetology on May 11th, 2020. See the published version at https://doi.org/10.1186/s12933-020-01033-4. 


\section{Abstract}

Background: To assess the prognostic role of coronary collaterals in patients with type 2 diabetes mellitus (T2DM) after successful percutaneous coronary intervention (PCl) for chronic total occlusion (CTO).

Methods: Coronary collateralization was graded according to Rentrop scoring system in 198 type 2 diabetic patients and 335 non-diabetics with stable angina undergoing PCl for at least one CTO lesion. Left ventricular ejection fraction (LVEF) was determined and major adverse cardio-cerebral events (MACCE) were recorded during follow-up.

Results: Poor collateralization was more common in patients with T2DM than in non-diabetics (40\% vs $29 \%, p=0.008)$. At $13.5 \pm 4.1$ months, the rate of composite MACCE ( $17.3 \%$ vs $27.6 \%, p=0.034)$ and repeat revascularization $(15.2 \%$ vs $25.5 \%, p=0.026)$ was lower and the increase in LVEF $(3.10 \%$ vs $1.80 \%$, $p=0.024)$ was greater in patients with good collaterals than in those with poor collaterals for non-diabetic group, but did not differ for T2DM group (35\% vs $44 \% ; 30 \%$ vs $36 \% ; 2.14 \%$ vs $1.65 \%$, respectively, all $p>0.05)$ except for a higher all-cause mortality in diabetic patients with poor collaterals $(p=0.034)$. Multivariable Cox proportional hazards analysis showed that coronary collateralization was an independent factor for time to MACCE (HR 2.155ه95\% Cl 1.290-3.599, $\mathrm{P}=0.003)$ and repeat revascularization (HR 2.326, 95\% Cl 1.357-3.986, $\mathrm{p}=0.002)$ in non-diabetic patients, but did not enter the model in those with T2DM.

Conclusions: T2DM is associated with reduced coronary collateralization. Successful revascularization of CTO lesions results in a mild improvement in left ventricular function, but the status of coronary collaterals has no independent effect on long-term clinical outcomes in patients with T2DM.

\section{Background}

Chronic total occlusion (CTO) occurs in $20-30 \%$ of patients with significant coronary artery disease undergoing routine coronary angiography ${ }^{1,2}$. Both randomized trials and observational studies have demonstrated that successful revascularization of chronic totally occluded lesions accomplished by percutaneous coronary intervention ( $\mathrm{PCl}$ ) or coronary artery bypass grafting is associated with a number of clinical benefits, such as anginal symptom relief, improved quality of life and ventricular function, and decreased mortality when compared to СTO patients whose recanalization was failed or those who received optimal medical treatment only ${ }^{3-6}$. Therefore, revascularization is recommended as an initial therapeutic modality in patients with CTO by current guidelines ${ }^{7}$. Recently, with the improvement in dedicated devices, technical strategies and interventional skills, the overall success rate of PCI for CTO (CTO-PCI) has been dramatically increased ${ }^{8}$.

Diabetes mellitus is regarded as a coronary heart disease risk equivalent and an important factor when planning treatment strategies for coronary artery disease as well as evaluating clinical outcomes after 
$\mathrm{PCl}{ }^{9,10}$. Patients with type 2 diabetes mellitus (T2DM) often have an elevated incidence of CTO (approximately $30 \%-40 \%$ in the registry) ${ }^{11,12}$. In the setting of complete coronary obstruction, blood supply to the distal myocardium is solely from collateral vessels. The formation and maturation of coronary collateral circulation is an adaptive physiologic response, and well-formed collaterals may minimize infarcted area and improve ventricular function and survival compared with patients lacking a well-developed collateral network ${ }^{13,14}$. However, the prognostic role of collaterals in patients with coronary artery disease remains controversial. In myocardial infarction patients with acute coronary occlusion, previous studies yielded mixed results with some showing improved clinical outcomes in patients with angiographic evidence of collaterals ${ }^{15-17}$ and others revealing either no difference ${ }^{18-20}$ or worse outcomes ${ }^{21}$. In stable coronary artery disease patients with CTO, while T2DM is strongly associated with reduced coronary collateral formation ${ }^{22-24}$, few published studies have focused on the impact of coronary collaterals on clinical prognosis, especially for those with T2DM after CTO-PCI ${ }^{6}$.

Therefore, we conducted a prospective, observational study to examine the effect of coronary collateralization on long-term clinical outcomes in type 2 diabetic and non-diabetic patients with stable coronary artery disease after successful CTO-PCI.

\section{Methods}

The protocol was approved by the Shanghai Jiao Tong University ethic committee and conducted in accordance with the Declaration of Helsinki. All patients gave written informed consent.

\section{Study population}

A total of 779 consecutive patients with stable angina who were attempted to undergo CTO-PCl of at least one major epicardial coronary artery between January 2016 and December 2018 were recruited from the database of Shanghai Rui Jin Hospital PCI Outcome Program. This program utilizes clinical and angiographic information for various cardiovascular diseases to estimate risk-adjusted outcomes. Data on demographics, clinical and angiographic features, left ventricular function determined by twodimensional echocardiography according to modified Simpson's rule, and in-hospital management were collected retrospectively, whereas clinical outcomes during follow-up were identified prospectively.

For the purpose of this study, 98 patients were excluded because of a history of coronary artery bypass grafting $(n=56)$, renal failure requiring hemodialysis $(n=12)$, chronic heart failure with NYHA class III or IV $(n=17)$, pulmonary heart disease $(n=6)$ and malignant tumor or immune system disorders $(n=7)$, as these conditions could influence collateral formation. Patients with type 1 diabetes $(n=6)$ were excluded by measurement of C-peptide level. In the 675 eligible patients, CTO-PCI was successful in 561 patients (83\%). The main causes for failed procedure included impossibility of wire $(n=85)$ or balloon $(n=15)$ to cross the occluded segment and major complications (coronary dissection: $n=9$; coronary perforation: 
$\mathrm{n}=5)$. We also excluded additional $28(5 \%)$ patients who were lost to follow up. Thus, the remaining 533 patients were enrolled in the final analyses. Among them, 198 patients (37\%) had T2DM and 335 (63\%) were non-diabetics (Figure 1).

CTO was defined as those occluded arteries with a documented duration of occlusion of at least 3 months with absolutely no flow through the lesion (TIMI grade 0$)^{25}$. Estimation of the duration of coronary occlusion was based on the first onset of an abrupt worsening of existing angina, a history of myocardial infarction in the target vessel territory, or information obtained from a previous angiogram. Stable angina was diagnosed according to the criteria recommended by the American College of Cardiology/ American Heart Association ${ }^{26}$. T2DM was defined as a fasting glucose level $>126 \mathrm{mg} / \mathrm{dL}$ or glycated hemoglobin A1c concentration greater than $6.5 \%$ assessed at least once, or the current use of oral hypoglycemic agents or insulin 27 .

\section{Coronary intervention procedure}

Coronary angiography and intervention were performed with standard techniques using $6 \mathrm{~F}$ or $7 \mathrm{~F}$ guiding catheters via the radial or femoral approach and drug-eluting stent implantation as the default strategy ( $>95 \%$ cases). Before the procedure, all patients received loading dose of aspirin $(300 \mathrm{mg} / \mathrm{d}$ ) and clopidogrel $(300 \mathrm{mg})$ or ticagrelor $(180 \mathrm{mg})$. During the procedure, an intravenous bolus of heparin (70-100 $\mathrm{IU} / \mathrm{kg}$ ) was given, but the use of glycoprotein Ilb/Illa inhibitors was at the operator's discretion. CTO-PCI was performed using contemporary techniques such as bilateral injection; specialized hydrophilic, tapered tip, and stiff wires; parallel wires; microcatheters; and retrograde approach. The choice of guidewires, balloons, and drug-eluting stent type was left to the discretion of the operators. After the procedure, clopidogrel $(75 \mathrm{mg} /$ day) or ticagrelor (90mg, twice daily) was prescribed for at least 12 months, and aspirin $(100 \mathrm{mg} /$ day) was continued indefinitely. After discharge, all patients were encouraged to take guideline- recommended medications including statins, angiotensin-converting enzyme inhibitors and $\beta$-blockers unless contraindicated, and to receive repeat coronary angiography at 12 months during follow-up.

Technical success was defined as a residual stenosis of $<20 \%$ and restoration of TIMI grade 3 flow. Procedural success was defined as technical success without death, myocardial infarction, or emergency coronary bypass grafting. Complete revascularization was defined as restoration of TIMI grade 3 flow with residual stenosis $<20 \%$ in all three major coronary arteries and their branches (diameter $\geq 2.0 \mathrm{~mm}$ ).

\section{Coronary collateral scoring}

The degree of coronary collaterals supplying the distal aspect of a total occlusion from the contra-lateral vessel was graded according to Rentrop classification: $0=$ no visible filling of any collateral channel; $1=$ 
filling of side branches of the artery to be perfused by collateral vessels without visualization of epicardial segment; 2 = partially filling of the epicardial artery by collateral vessels; $3=$ complete filling of the epicardial artery by collateral vessels ${ }^{28}$. Patients were categorized into poor (grade 0 or 1 ) or good (grade 2 or 3 ) coronary collateralization group. All angiograms were viewed by the two observers blinded to the other observers' findings, and the agreement of the assessment of coronary artery disease severity and collateral classification between the two observers was $98 \%$ and $97 \%$, respectively ${ }^{29}$. Any difference in interpretation was resolved by a third reviewer. For those with more than one total coronary occlusion, the vessel with the highest collateral grade was chosen for analysis.

\section{Study endpoints}

The primary study endpoint was the occurrence of composite major adverse cardio-cerebral events (MACCE) during follow-up, including all-cause death, cardiac death, non-fatal myocardial infarction, repeat revascularization, and non-fatal stroke. All-cause death was defined as any post-procedure death, and the cause was considered cardiac unless a definite non-cardiac cause was established. Myocardial infarction was defined as recurrent symptoms with new electrocardiographic changes compatible with myocardial infarction or cardiac marker level at least twice the upper limit of normal. Repeat revascularization was defined as any revascularization of either the target or non-target vessels with $\mathrm{PCl}$ or coronary artery bypass grafting. In-stent restenosis was defined as recurrence of lumen diameter reduction $>50 \%$ within the stent or $5 \mathrm{~mm}$ proximal or distal segment adjacent to the stent at follow-up angiography ${ }^{30}$. Atherosclerotic lesion progression was diagnosed if one of the following criteria was met: (1) $\geq 20 \%$ diameter reduction of a pre-existing stenosis $>50 \%$; (2) $\geq 30 \%$ diameter reduction of a stenosis $<50 \%$; (3) progression of any stenosis to total occlusion, or (4) development of a new stenosis $>50 \%$ in a previously normal segment ${ }^{31}$. The secondary study endpoint was the change in left ventricular ejection fraction (LVEF) determined by two-dimensional echocardiography using modified Simpson's method.

\section{Statistical analysis}

Continuous variables are expressed as mean \pm standard deviation (SD) and categorical data as percentages. Two-side Student's t test was used to compare continuous variables, and Pearson chisquare statistics was used to compare categorical values. The rate of composite MACCE and repeat revascularization were compared by calculating risk ratio with $95 \%$ confidence intervals (Cls). Cumulative rate of individual and composite outcomes was estimated using the Kaplan-Meier methods and compared with the log-rank test. Multivariable models were built by stepwise variable selection, and covariates with $p<0.10$ level on univariable analysis or clinically relevant were considered candidate variables. Adjusted hazard ratios were compared by Cox regression based on: age, gender, risk factors for coronary artery disease (current smoking, hypertension, hypercholesterolemia, and diabetes), extent of coronary artery disease (categorized as 1-, 2-, or 3-vessel disease), collateral classification, glomerular 
filtration rate, pre-procedural LVEF, and completeness of revascularization. A probability level of $p<0.05$ was considered significant. All analyses were performed using the software package SPSS, version 13 (SPSS Inc, Chicago, IL, USA).

\section{Results}

\section{Clinical and angiographic features}

Compared with non-diabetic patients, those with T2DM were more males in gender distribution, and had higher percentage of hypertension, multivessel disease and poorer coronary collaterals, and lower LVEF (Table 1). Univariable regression analysis showed that presence of T2DM was the only determinant of poor coronary collateralization $(r=0.114, p=0.008)$.

During PCl procedure, a total of 560 CTO lesions were successfully recanalized with drug-eluting stent implantation. There were no differences in location of CTO, stent number for CTO lesion and the rate of complete revascularization between patients with and without T2DM except that those with T2DM had higher proportion of multivessel disease $(p=0.006)$ (Table 1).

\section{Long-term clinical outcomes}

During a mean of $13.5 \pm 4.1$ months of follow-up, 8 patients died (1.5\%); 5 of them had cardiac death (good collaterals in 1 patient [0.3\%] and poor collaterals in 4 patients [2.2\%], $p=0.026$ ). and other 3 died of respiratory failure due to pulmonary infection $(n=1)$ or cerebrovascular accidents $(n=2)$. Repeat revascularization was performed in 125 patients (23.5\%) because of in-stent restenosis or new lesions and disease progression. Overall, the rate of composite MACCE ( $38 \%$ vs $19.4 \%, p<0.001$ ) and repeat revascularization ( $32 \%$ vs $18.2 \%, p<0.001)$ was higher in type 2 diabetic patients compared to nondiabetics.

For non-diabetic patients, poor collateralization was associated with more in-stent restenosis ( $14.3 \% \mathrm{vs}$ $6.3 \%, p=0.018)$ and repeat revascularization $(25.5 \%$ vs $15.2 \%, p=0.026)$, leading to a higher rate of composite MACCE $(27.6 \%$ vs $17.3 \%, p=0.034)$. In contrast, for type 2 diabetic patients, the rate of composite MACCE, in-stent restenosis and repeat revascularization did not differ irrespective of collateral status, except for a higher all-cause mortality in those with poor collateralization $(p=0.034)$ (Table 2). Kaplan-Meier curve analysis revealed a clear difference in percent event-free from MACCE or repeat revascularization according to the presence or absence of T2DM or Rentrop class of coronary collateralization (Figure 2).

\section{Left ventricular function}


Baseline LVEF was higher in patients with good collaterals than that in those with poor collateral for nondiabetics but was similar between type 2 diabetic patients with good and poor collaterals. Successful CTO-PCI was associated with a mild increase in LVEF irrespective of the status of diabetes and coronary collateralization (all $p<0.001$ ). The degree of increase in LVEF was significantly greater in patients with good collaterals than in those with poor collaterals for non-diabetic group $(3.10 \%$ vs $1.80 \%, p=0.024)$ but did not differ between patients with good and poor collaterals for T2DM group (Table 3).

\section{Multivariate analysis}

Multivariable Cox proportional hazards regression analysis revealed that T2DM but not the status of coronary collateralization was independent factors for time to MACCE (HR 1.633 $85 \% \mathrm{Cl}$ 1.157-2.304, $\mathrm{P}=0.005)$ and repeat revascularization (HR 1.515, 95\% Cl 1.046-2.192, $\mathrm{p}=0.028)$. Further analysis showed that the status of coronary collateralization was an independent factor for time to MACCE (HR 2.155区 $95 \% \mathrm{Cl} 1.290-3.599, \mathrm{P}=0.003$ ) and repeat revascularization (HR 2.326, 95\% Cl 1.357-3.986, $\mathrm{p}=0.002$ ) in non-diabetic patients, but it did not enter the model in those with T2DM (Table 4).

\section{Discussion}

The results of this prospective, observational study show that: 1) T2DM was associated with reduced coronary collateralization; 2) successful revascularization of CTO lesions slightly improved left ventricular function; 3 ) the status of coronary collaterals exerted an independent effect on prognosis in non-diabetic patients but not in those with T2DM.

Notably, all patients in this study were specially selected as they received successful CTO-PCI and were encouraged to take guideline-recommended medications after procedure. Our findings support the notion that T2DM represents an increased risk for CTO 2,11,12 and is a powerful independent factor for increased cardiovascular mortality and repeat revascularization after $\mathrm{CTO}-\mathrm{PCl}{ }^{9,10}$. Abundant evidence has demonstrated that T2DM exerts a detrimental effect on glucose and lipid metabolism and vascular endothelial function, leading to development and progression of coronary atherosclerosis and unfavorable clinical outcomes ${ }^{32-35}$. In this study, T2DM was the only independent risk factor for poor coronary collateralization, suggesting that presence of T2DM is correlated negatively with the development of functional collateral arteries ${ }^{22,23}$, and may contribute partially to adverse prognosis of СTO patients ${ }^{36}$. Although presence of a chronic totally occluded lesion has been considered as a prerequisite for spontaneous collateral recruitment, the mechanism of collateral vessel growth is complex in situations where atherosclerosis affects large conductance arteries ${ }^{37}$, and even become more complicated by the presence of T2DM in which multiple biochemical and cellular components are involved $^{23,38,39}$. 
Another finding of this study is that around one-year post successful CTO-PCl, although patients with good coronary collateralization experienced a significantly lower rate of composite MACCE and repeat revascularization compared to those with poor collateralization, and Kaplan-Meier curves revealed the cumulative survival free from repeat revascularization and MACCE was significantly different according to the Rentrop class of coronary collateralization, multivariable Cox proportional hazards regression models revealed that the status of coronary collateralization was independently associated with longterm clinical outcomes only in non-diabetic patients but not in those with T2DM. The explanation for these observations is likely to be multi-factorial. Numerous studies have shown that when the proximal part of a coronary artery is occluded, collateral circulation could, at least partially, supplies the downstream perfusion area via the arteriolar connection, thereby preventing or alleviating ischemia, and its extent is a primary determinant of the severity of myocardial damage (infarct size and/or left ventricular function) and mortality after transient or permanent coronary obstruction ${ }^{13,14}$. However, blood supply of the well-developed collaterals may not fully substitute normal coronary flow ${ }^{1,40}$, and thus good collateral circulation for myocardial protection is not sufficient. Importantly, good collaterals in majority of patients will be rapidly subside after successful recanalization of a CTO, as antegrade blood flow is reestablished and resistance is increased in the collateral vessels ${ }^{36,40}$. Kim et al found that good collateral circulation for myocardial protection only exists before recanalization forms an effective perfusion ${ }^{41}$. In addition, previous studies suggest that the occurrence of in-stent restenosis after successful CTO-PCl is not influenced by coronary collateralization ${ }^{30}$ or even elevated in patients with good collaterals ${ }^{42}$. These could lead to a similar or increased rate of repeat revascularization and overall MACCE in patients with good collaterals after success CTO-PCl.

In this study, mean LVEF was slightly increased by $2.50 \%$ during follow-up which is lower than that previously reported $(4.44 \%){ }^{43}$. Nevertheless, non-diabetic patients with good collateralization had a significantly greater improvement in left ventricular function compared to those with poor collaterals. Ripley et al found that viable myocardium was present in $83 \%$ of patients with good collaterals versus $38 \%$ of those with poor collaterals ${ }^{44}$. Likewise, Choi et al observed that increased angiographic collateral flow was associated with lower degree of late gadolinium enhancement transmurality after CTO recanalization, in contrast, poorly collateralized myocardial segments would be less likely to recover function in comparison with well collateralized segments ${ }^{45}$. Compared with non-diabetics, type 2 diabetic patients had lower LVEF at baseline, and the change in LVEF was smaller and did not differ after successful CTO-PCI irrespective of collateral status. These findings are consistent with previous reports that myocardial and collateral function is more severely depressed in the diabetic setting $9,10,22,23$.

\section{Study limitations}

We recognize that there are several limitations in our study. First, a potential weakness of our study is the small sample size and heterogeneity with respect to the patient distribution. Second, the study is cross- 
sectional for the point of coronary collateral and MACCE or improvement of LVEF investigation, thereby allowing us to detect association, not to formulate causal link. Third, the presence and degree of coronary collaterals were evaluated according to the Rentrop scoring system. Although this angiographic assessment of coronary collaterals is easily to be incorporated into the routine clinical practice, coronary collaterals may be more accurately assessed by collateral flow index with simultaneous measurement of aortic pressure and the distal pressure within the occluded segment of the culprit coronary artery 40 . Finally, clinical follow-up was relatively short in duration, and amount of viable myocardium or ischemia was not evaluated using functional tests, thus large-scale prospective studies are required to further determine the impact of coronary collateral circulation on prognosis after successful CTO-PCI.

\section{Conclusions}

The present study suggests that presence of T2DM is associated with reduced coronary collateralization. Successful revascularization of CTO lesions results in a mild improvement in left ventricular function, but the status of coronary collaterals has no independent effect on long-term clinical outcomes in type 2 diabetic patients. These findings may provide clinical insight into the management of patients with stable coronary artery disease.

\section{Abbreviations}

CTO: chronic total occlusion; HR: hazardous ratio; LVEF: left ventricular ejection fraction; MACCE: major adverse cardiac and cerebral events; NYHA: New York Heart Association; PCl: percutaneous coronary intervention; T2DM: type 2 diabetes mellitus; SD: standard deviation.

\section{Declarations}

\section{Ethics approval and consent to participate}

The study protocol was approved by the Institutional Review Board of Rui Jin Hospital, Shanghai Jiaotong University School of Medicine. Written informed consent was obtained from all patients, and clinical investigation was conducted according to the principle of the Declaration of Helsinki.

\section{Consent for publication}

All authors consent this manuscript for publication.

\section{Availability of data and materials}

Data generated or analyzed during this study are included in this published article. 


\section{Competing interests}

The authors declare that they have no competing interests.

\section{Funding}

This study was supported in part by the Research Foundation of Chinese National Natural Science (81970362, 81970293, 81870357), Talent Young Investigators (17XJ11009) of Shanghai Jiao Tong University School of Medicine and Shanghai Municipal Education Commission-Gaofeng Clinical Medicine Grant Support (20181801).

\section{Authors' contributions}

ZKY, YS, WFS wrote the article, substantially contributed to discussion of the content, and edited the manuscript. ZKY, YS, YD, XQW, JH®FHD performed the experiments and researched data for the article. ZKY analyze the data; LL, RYZ substantially contributed to discussion of the content and reviewed the manuscript.

\section{Acknowledgements}

Not applicable.

\section{Author details}

1 Department of Cardiology, Rui Jin Hospital, Shanghai Jiao Tong University School of Medicine, Shanghai 200025, People's Republic of China.

2 Institute of Cardiovascular Disease, Shanghai Jiao Tong University School of Medicine, 197 Rui Jin Road II, Shanghai 200025, People's Republic of China.

Authors' email address: Zhen Kun Yang (yangzhenkun2002@hotmail.com); Ying Shen (rjshenying@qq.com); Yang Dai (yutongwushe@163.com); Xiao Qun Wang (xiaoqun_wang@hotmail.com); Jian Hu (hujian@medmail.com,cn); Feng Hua Ding (ruijindfh@126.com); Ruin Yan Zhang (zhangruiyan@263.net) ;Lin L(rjlulin1965@163.com);Wei Feng Shen (rjshenweifeng@126.com) 


\section{References}

1. Werner GS. The role of coronary collaterals in chronic total occlusion. Curr Cardiol Rev. 2014; 10:5764.

2. Fefer P, Knudtson ML, Cheema AN, Galbraith PD, Osherov AB, Yalonetsky S, et al. Current perspectives on coronary chronic total occlusions: The Canadian Multicenter Chronic Total Occlusions Registry. J Am Coll Cardiol. 2012; 59: 991-7

3. Werner GS, Martin-Yuste V, Hildick-Smith D, Boudou N, Sianos G, Gelev V, et al. EUROCTO trial investigators. A randomized multicentre trial to compare revascularization with optimal medical therapy for the treatment of chronic total coronary occlusions. Eur Heart J. 2018;39:2484-2493.

4. Jang WJ, Yang JH, Choi SH, Song YB, Hahn JY, Choi JH, et al. Long-term survival benefit of revascularization compared with medical therapy in patients with coronary chronic total occlusion and well-developed collateral circulation. JACC Cardiovasc Interv. 2015; 8:271-9.

5. Gao L, Wang Y, Liu Y, Cao F, Chen Y. Long-term clinical outcomes of successful revascularization with drug-eluting stents for chronic total occlusions: A systematic review and meta-analysis. Cathet Cardiovasc Interv. 2017; 89:574-81.

6. Choi KH, Yang JH, Song YB, Hahn JY, Choi JH, Gwon HC, et al. Long-term outcomes of patients with coronary chronic total occlusion treated with percutaneous coronary intervention versus medical therapy according to presence of diabetes mellitus. Eurointervention 2017; 13:970-7.

7. Neumann FJ, Sousa-Uva M, Ahlsson A, Alfonso F, Banning AP, Benedetto U, et al. 2018 ESC/EACTS Guidelines on myocardial revascularization. Eur Heart J 2019; 40:87-165.

8. Tajti P, Burke MN, Karmpaliotis D, Alaswad K, Werner GS, Azzalini L, et al. Update in the percutaneous management of coronary chronic total occlusions. JACC Cardiovasc Interv 2018; 11:615-25.

9. Wang Q, Liu H, Ding J. Outcomes of percutaneous coronary intervention in patients with coronary chronic total occlusions with versus without type 2 diabetes mellitus: A systematic review and metaanalysis. Medicine (Baltimore) 2017; 96(45): e8499.

10. Mashaly A, Rha SW, Choi BG, Baek MJ, Ryu YG, Choi SY, et al. Impact of diabetes mellitus on 5-year clinical outcomes in patients with chronic total occlusion lesions. Coron Artery Dis. 2018; 29:119-26.

11. Michael TT, Karmpaliotis D, Brilakis ES, Fuh E, Patel VG, Mogabgab O, et al. Procedural outcomes of revascularization of chronic total occlusion of native coronary arteries (from a multicenter United States registry). Am J Cardiol. 2013; 112:488-92.

12. Brilakis ES, Banerjee S, Karmpaliotis D, Lombardi WL, Tsai TT, Shunk KA, et al. Procedural outcomes of chronic total occlusion percutaneous coronary intervention: a report from the NCDR (National Cardiovascular Data Registry). JACC Cardiovasc Interv. 2015; 8:245-53.

13. Meier P, Hemingway H, Lansky AJ, Knapp G, Pitt B, Seiler C. The impact of the coronary collateral circulation on mortality: a meta-analysis. Eur Heart. J 2012; 33:614-21.

14. Regieli JJ, Jukema JW, Nathoe HM, Zwinderman AH, Ng S, Grobbee DE, et al. Coronary collaterals improve prognosis in patients with ischemic heart disease. Int J Cardiol. 2009; 132:257-62. 
15. Monteiro P, Antunes A, Goncalves LM, Providencia LA. Long-term clinical impact of coronary collateral vessels after acute myocardial infarction. Rev Port Cardiol. 2003; 22:1051-61.

16. Elsman P, van 't Hof AW, de Boer MJ, Hoorntje JC, Suryapranata H, Dambrink JH, Zijlstra F; Zwolle Myocardial Infarction Study Group. Role of collateral circulation in the acute phase of ST-segmentelevation myocardial infarction treated with primary coronary intervention. Eur Heart J. 2004; 25:854-8.

17. Desch S, de Waha S, Eitel I, Koch A, Gutberlet M, Schuler G, et al. Effect of coronary collaterals on long-term prognosis in patients undergoing primary angioplasty for acute ST-elevation myocardial infarction. Am J Cardiol. 2010; 106:605-11.

18. Sorajja P, Gersh BJ, Mehran R, Lansky AJ, Krucoff MW, Webb J, et al. Impact of collateral flow on myocardial reperfusion and infarct size in patients undergoing primary angioplasty for acute myocardial infarction. Am Heart J. 2007; 154:379-84.

19. Steg PG, Kerner A, Mancini GB, Reynolds HR, Carvalho AC, Fridrich V, et al; OAT Investigators. Impact of collateral flow to the occluded infarct-related artery on clinical outcomes in patients with recent myocardial infarction: a report from the randomized occluded artery trial. Circulation. 2010; 121:2724-30.

20. Shen Y, Wu F, Pan C, Zhu T, Zhang Q, Zhang R, et al. Clinical relevance of angiographic coronary collaterals during primary coronary intervention for acute ST-elevation myocardial infarction. Chin Med J (Engl) 2014; 127:66-71

21. Nicolau JC, Nogueira PR, Serrano CV Jr, Garzon SA. Early infarct artery collateral flow does not improve long-term survival following thrombolytic therapy for acute myocardial infarction. Am $J$ Cardiol. 1999; 1:83:21-6.

22. Shen Y, Lu L, Ding FH, Sun Z, Zhang RY, Zhang Q, et al. Association of increased serum glycated albumin levels with low coronary collateralization in type 2 diabetic patients with stable angina and CTO. Cardiovasc Diabetol. 2013; 12:165.

23. Shen Y, Ding FH, Dai Y, Wang XQ, Zhang RY, Lu L, et al. Reduced coronary collateralization in type 2 diabetic patients with chronic total occlusion. Cardiovasc Diabetol. 2018; 17:26.

24. Yetkin E, Topal E, Erguzel N, Senen K, Heper G, Waltenberger J. Diabetes mellitus and female gender are the strongest predictors of poor collateral vessel development in patients with severe coronary artery stenosis. Angiogenesis. 2015; 18:201-7.

25. Galassi AR, Werner GS, Boukhris M, Azzalini L, Mashayekhi K, Carlino M, et al. Percutaneous recanalisation of chronic total occlusions: 2019 consensus document from the EuroCTO Club. Eurolntervention. 2019; 15:198-208.

26. Fraker TD, Jr., Fihn SD, Gibbons RJ, Abrams J, Chatterjee K, Daley J, et al. 2007 chronic angina focused update of the ACC/AHA 2002 Guidelines for the management of patients with chronic stable angina: a report of the American College of Cardiology/American Heart Association Task Force on Practice Guidelines Writing Group to develop the focused update of the 2002 Guidelines for the management of patients with chronic stable angina. Circulation. 2007; 116:2762-72. 
27. American Diabetic Association. Diagnosis and classification of diabetes mellitus. Diabetes Care. 2013; 36 (Supplement 1): S67-S74.

28. Rentrop KP, Cohen M, Blanke H, Phillips RA. Changes in collateral channel filling immediately after controlled coronary artery occlusion by an angioplasty balloon in human subjects. J Am Coll Cardiol. 1985; 5:587-92.

29. Shen Y, Ding FH, Zhang RY, Zhang Q, Lu L, Shen WF. Association of serum mimecan with angiographic coronary collateralization in patients with stable coronary artery disease and chronic total occlusion. Atherosclerosis. 2016囚252:75-81.

30. Yang ZK, Shen Y, Hu J, Zhang Q, Ding FH, Zhang RY,et al. Impact of coronary collateral circulation on angiographic in-stent restenosis in patients with stable coronary artery disease and chonic total occlusion. Int J Cardiol. 2017; 227:485-.9.

31. Pu LJ. Lu L, Zhang RY, Du R, Shen Y, Zhang Q, et al. Glycation of apoprotein A-1 is associated with coronary artery plaque progression in type 2 diabetes mellitus. Diabetes Care 2013; 36:1312-20.

32. Yang ZK, Shen Y, Shen WF, Pu L, Meng H, Zhang RY, et al. Elevated glycated albumin and reduced endogenous soluble receptors for advanced glycation endproducts in serum predict major advanced cardio-cerebral events in patients with type 2 diabetes and stable coronary artery disease. Int $\mathrm{J}$ Cardiol. 2015; 197:241-7.

33. Du R, Zhang RY, Lu L, Shen Y, Pu L, Zhu ZB, et al. Increased glycated albumin and decreased esRAGE in serum are related to negative coronary artery remodeling in patients with type 2 diabetes: an intravascular ultrasound study. Cardiovasc Diabetol. 2018; 17:149.

34. Shen Y, Ding FH, Sun Z, Pu L, Zhang RY, Zhang Q, et al. Association of elevated apoA-1 glycation and reduced paraoxonase 1, 3 activity, and their interaction with angiographic severity of coronary artery disease in patients with type 2 diabetes, Cardiovasc Diabetol. 2015; 14:52.

35. Dai Y, Shen Y, Li QR, Ding FH, Wang XQ, Liu HJ, et al. Glycated apolipoprotein A-IV induces atherosclerosis in patients with CAD in type 2 diabetes. JACC 2017; 70:206-19.

36. Werner GS, Richartz BM, Heinke S, Ferrari M, Figulla HR. Impaired acute collateral recruitment as a possible mechanism for increased cardiac adverse events in patients with diabetes mellitus. Eur Heart J. 2003; 24(12):1134-42.

37. Zimarino M, D'Andreamatteo M, Waksman R, Epstein SE, De Caterina R. The dynamics of the coronary collateral circulation. Nat Rev Cardiol. 2014; 11:191-7.

38. Shen Y, Chen S, Dai Y, Wang XQ, Zhang RY, Yang ZK, et al. Lpoprotein (a) interactions with cholesterol-containing lipids on angiographic coronary collateralization in type 2 diabetic patients with chronic total occlusion. Cardiovasc Diabetol. 2019; 18:82.

39. Shen Y, Dai Y, Wang XQ, Zhang RY, Lu L, Ding FH, et al. Searching for optimal blood pressure targets in type 2 diabetic patients with coronary artery disease. Cardiovasc Diabetol. 2019; 18:160.

40. Shen Y, Yang ZK, Hu J, Wang XQ, Dai Y, Zhang S, et al. Donor artery stenosis interactions with diastolic blood pressure on coronary collateral flow in type 2 diabetic patients with chronic total occlusion. Cardiovasc Diabetol. 2018; 17:76. 
41. Kim BM, Baek JH, Heo JH, Nam HS, Kim YD, Yoo J, et al. Collateral status affects the onset-toreperfusion time window for good outcome. J Neurol Neurosurg Psychiatry. 2018; 89:903-.9.

42. Meier P, Indermuehle A, Pitt B, Traupe T, de Marchi SF, Crake T, et al. Coronary collaterals and risk for restenosis after percutaneous coronary intervention: a meta-analysis. BMC Med. 2012; 10:62-73.

43. Hoebers LP, Claessen BE, Elias J, Dangas GD, Mehran R, Henriques JP. Meta-analysis on the impact of percutaneous coronary intervention of chronic total occlusions on left ventricular function and clinical outcome. Int J Cardiol. 2015; 187:90-6.

44. Ripley DP, Gosling OE, Bhatia L, Peebles CR, Shore AC, Curzen N, et al. The relationship between the contralateral collateral supply and myocardial viability on cardiovascular magnetic resonance: can the angiogram predict functional recovery? Int J Cardiol. 2014; 177:362-7.

45. Choi JH, Chang SA, Choi JO, Song YB, Hahn JY, Choi SH, et al. Frequency of myocardial infarction and its relationship to angiographic collateral flow in territories supplied by chronically occluded coronary arteries. Circulation. 2013; 127:703-9.

\section{Tables}

Please see the supplementary files section to access the tables.

\section{Figures}




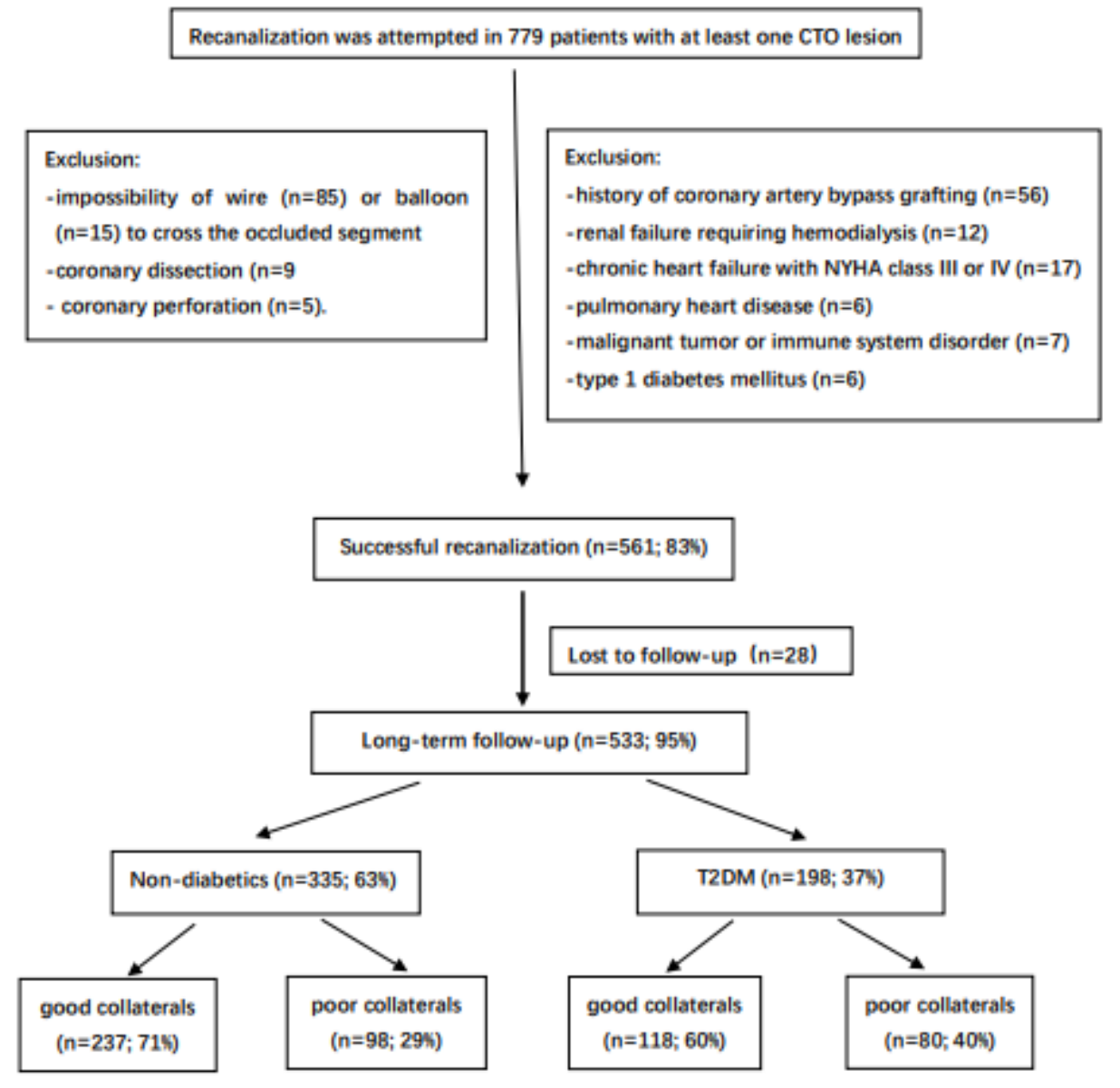

\section{Figure 1}

Flowchart patient enrollment. CTO: chronic total occlusion; T2DM: type 2 diabetes mellitus 

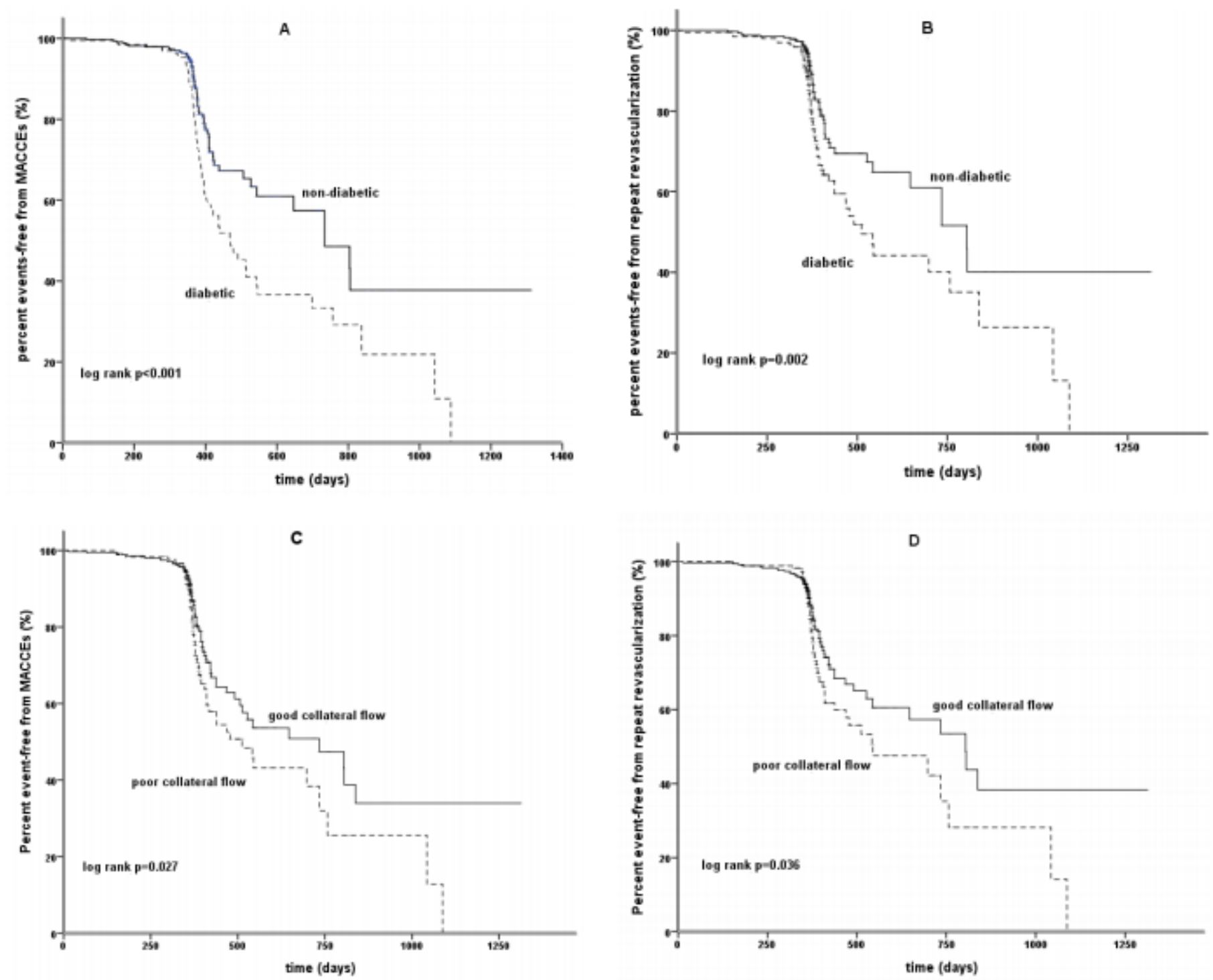

Figure 2

Kaplan-Meier curves. Percent event-free from MACCE and repeat revascularization in non-diabetic and type 2 diabetic patients ( $A$ and $B$ ) and in those with good and poor coronary collateral circulation ( $C$ and D). MACCE: major adverse cardio-cerebral events

\section{Supplementary Files}

This is a list of supplementary files associated with this preprint. Click to download.

- Table2.xlsx

- Table4.xlsx

- Table1.xlsx

- Table3.xlsx 